\title{
Switched Parasitic Patch Antenna Array Using Thirteen Hexagonal Shaped Elements
}

\author{
Junwei. Lu ${ }^{1}$, Anthony Stark ${ }^{2}$, David Thiel ${ }^{3}$ \\ Centre of Wireless Monitoring and Applications, Griffith University, Nathan, Brisbane, Qld 4111, Australia \\ ${ }^{1}$ j.ludgriffith.edu.au \\ ${ }^{2}$ a.starkegriffith.edu.au \\ ${ }^{3}$ d.thielegriffith.edu.au
}

\begin{abstract}
A novel circularly polarized smart patch antenna array with hexagonal elements was designed as a hub for indoor/outdoor mobile wireless computing networks. A frequency domain finite element method (FEM) was employed to design the patch antenna array using an optimization technique based on the gradient algorithm to optimize the physical structure on a finite ground plane for optimal directivity in elevation plane and antenna gain at $2.4 \mathrm{GHz}$. The switched parasitic element technique was used to enable steering through six locations in azimuth with an elevation angle of between $60^{\circ}$ and $70^{\circ}$. The single-feed circularly polarized patch antenna array with thirteen-element configuration achieved the highest frequency bandwidth. A comparison between simulated and measured reflection coefficients and radiation patterns was made with consistent results.
\end{abstract}

\section{INTRODUCTION}

The smart antennas for mobile wireless communications has received significant interest world-wide in last decade. However, the application of smart antennas in mobile communication and wireless computing network terminals has received little attention due to the complexity of the antenna structure, size reduction techniques, and the system constraints associated with the antenna technology. In recent years, a number of smart mobile terminal antennas (SMTAs) have been developed. Among them, Schlub's seven-element ground skirt monopole antenna array based on electronically steerable passive array radiator (ESPAR) for wireless ad hoc computer networks [1] and Lu's dielectric embedded ESPAR antenna array for mobile terminals [2] operating at $2.4 \mathrm{GHz}$ have been successfully developed for mobile wireless computing network, Gray's switched Yagi patch antenna for mobile satellite communication [3] and Thiel's beam-switched monopole arrays [4] have also been explored for the application in wireless communications and computing network systems. However, the antenna array as an access point mounted in the ceiling and facing down to computer users for indoor/outer door mobile wireless computing has yet to be fully reported, except for some previous work on a patch antenna with seven hexagonal element (PASHE) antenna array developed by Shi [5].

Unlike traditional antennas used in current wireless computing network system, the newly developed smart patch antenna arrays can significantly increase antenna gain and the wireless security level in WLAN systems at $2.4 \mathrm{GHz}$ by using computer controlled switched beam or adaptive beam-forming technologies. This smart antenna can switch the beam to one of six, evenly-spaced directions in the azimuth plane. The antenna can therefore track all of the mobile wireless computing terminals in the area. Figure 1 shows a typical application of the smart patch antenna mounted in the ceiling as an access point for indoor/outer door WLAN applications with sectorized beam coverage for desired direction only. Obviously the bester performance will be achieved when the cluster users exist, and it is not suitable for loosely distributed user group.

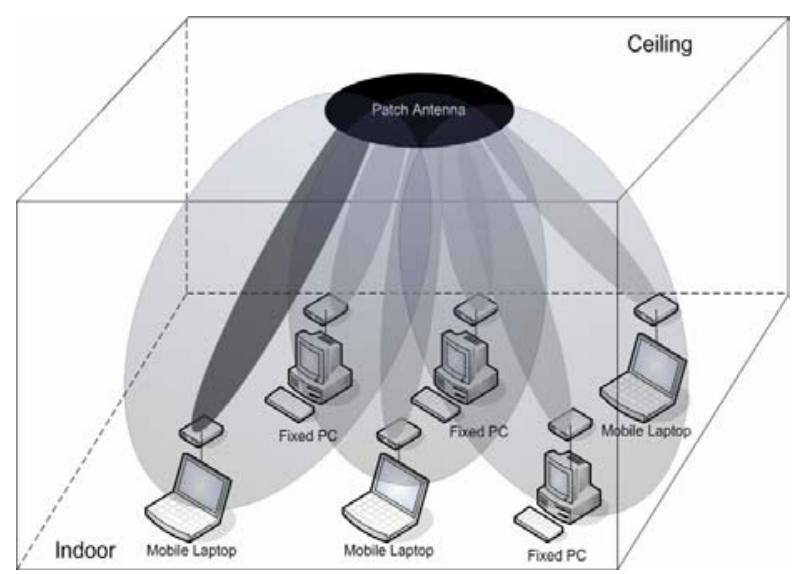

(a) Antenna mounted on the ceiling for indoor wireless network system

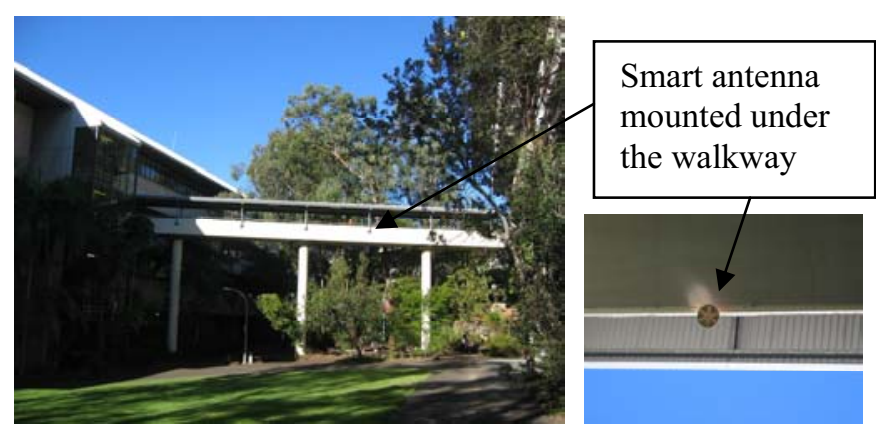

(b) Antenna mounted under the walkway for outdoor wireless network system

Fig.1 An access point for indoor/outdoor WLAN system using smart antenna. 978-1-4244-2193-0/08/\$25.00 @2008 IEEE 


\section{ThiRTEEN HEXIGONAL ELEMENT ANTENNA ARRAY}

\section{A. Antenna Configuration}

In previous designs, two feeds were used for the driven element patch to generate circular polarization for better reception and wave propagation performance [5]. Since this antenna array requires two sources for driven element and more additional RF components, a narrow rectangular slot placed in the central driven element [6] as shown in Fig. 2 (a) was selected to provide circular polarization. The slot was cut into the active patch element, removing the need for a more complicated dual feed system. To increase antenna gain at each direction and reduce elevation angle, the single-feed Patch Antenna with Thirteen Hexagonal Elements (PATHE) was further developed as shown in Fig. 2 (b), where the central element is a driven element surrounded by six equal size and shape elements. The additional elements with same orientation placed at outer circle were used as directors, while the inner circle of six switched parasitic elements or reactively loaded parasitic elements remain in the same configuration as PASHE [5] The frequency domain FEM based simulator, HFSS [7] was employed to design and optimize the antenna performance and physical structure. Whilst the cavity model is unsuited to detailed analysis of the PATHE antenna, it does provide a useful starting point at which design work in HFSS $^{\text {TM }}$ can begin. A cavity model approximation was used to provide the radius for the parasitic patches. This is given by equation

$$
a=\frac{F}{\left\{1+\frac{200 h}{\pi \varepsilon_{r} F}\left[\ln \left(\frac{\pi F}{200 h}\right)+1.7726\right]\right\}^{1 / 2}}
$$

where

$$
F=\frac{8.791 \times 10^{9}}{f_{r} \sqrt{\varepsilon_{r}}}
$$

and $a, h, \varepsilon_{r}$ and $f_{\mathrm{r}}$ are radius of element, dielectric substrate height, relative permittivity of dielectric, and resonant frequency respectively.

Elements were approximated to circles with a dominant $\mathrm{TM}^{\mathrm{Z}}{ }_{110}$ mode. For a $1.6 \mathrm{~mm}$ FR4 epoxy substrate $\left(\varepsilon_{r}=4.5\right)$ and an operating frequency of $2.4 \mathrm{GHz}$, a $3.35 \mathrm{~cm}$ diameter patch was given. To properly terminate the induced circular currents, two orthogonal terminations were used for each of the switched elements. The termination points can be placed on either inner or outer patch element. These termination points do not affect the antenna performance.

All elements in the outer circle and one of elements in the inner circle function as director elements and so were opencircuited. The remaining elements in inner circle were shortcircuited to ground at five directions in azimuth plane. A typical beam-forming circuit for the single-feed circularly polarized patch antenna array, PATHE, using switched beam- forming structure is illustrated in Fig. 3 (a). The beam control circuit is much simpler than the one used in dual-feed structure as shown in Fig. 3 (b).
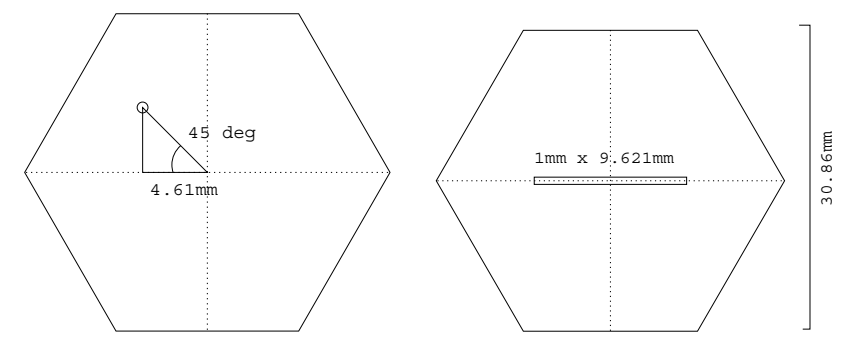

(a) single feed and a narrow rectangular slot

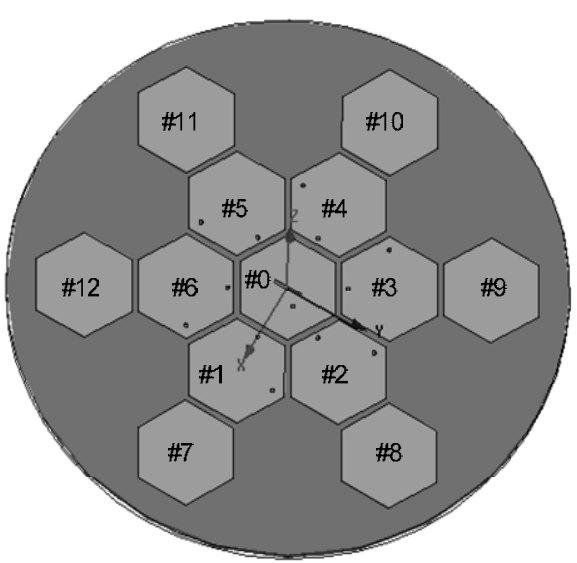

(b) Layout of patch antenna with thirteen hexagonal elements

Fig 2. The single-feed patch antenna with thirteen hexagonal elements

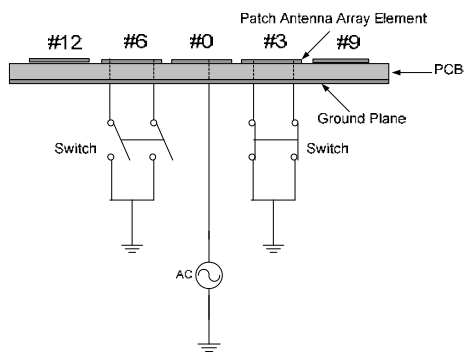

(a) dual-switch circuit for beam forming

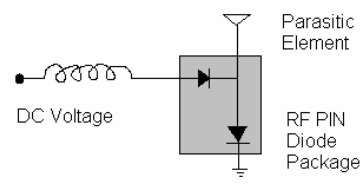

(b) $R F$ Pin diode switch
Fig. 3 A typical beam-forming circuit for the single-feed circularly polarized patch antenna array.

\section{B. Implementation}

Figure 4 shows a block diagram of the control circuit for the PATHE. The system consisted of four parts: the physical antenna, the switching circuitry, a USB controller and PC based software. A simple USB device was used to generate switching currents required for the antenna. Table I shows the switching table where six position can be performed by different switching combination. A switching current of $4 \mathrm{~mA}$ at 4.8 volts was used to switch each element. Signal feedback 
was introduced via an external wireless monitor. This allowed the PATHE antenna to dynamically track a given signal.

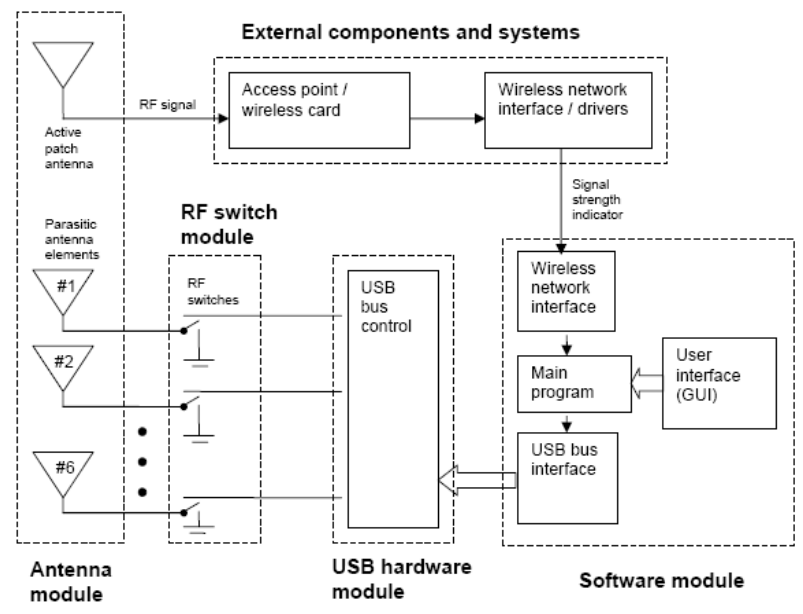

(a) The diagram of beam control circuit

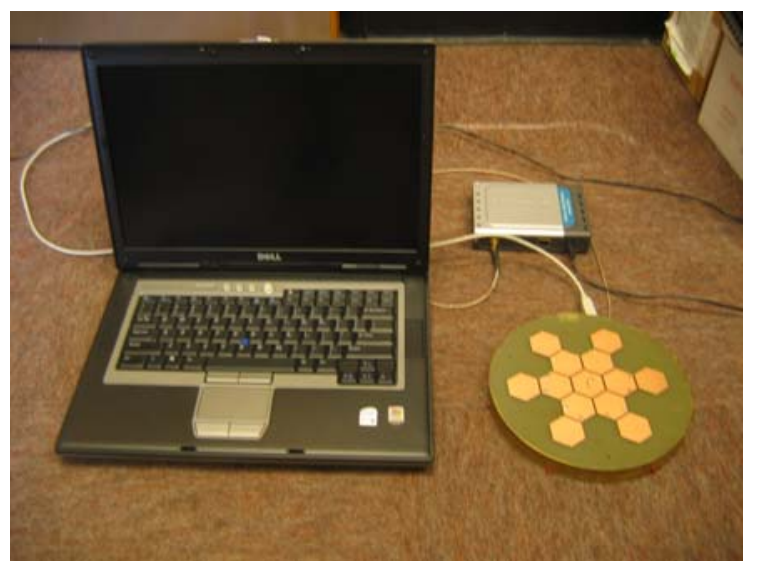

(b) System configuration of wireless terminal with assess point

Fig. 4 Smart antenna used in mobile wireless computing network

TABLE I

Beam Directions And Six Switching Positions

\begin{tabular}{|l|l|l|l|l|l|l|}
\hline Beam/Element & $\mathbf{1}$ & $\mathbf{2}$ & $\mathbf{3}$ & $\mathbf{4}$ & $\mathbf{5}$ & $\mathbf{6}$ \\
\hline 0 degree & Off & On & On & On & On & On \\
\hline 60 degree & On & Off & On & On & On & On \\
\hline 120 degree & On & On & Off & On & On & On \\
\hline 180 degree & On & On & On & Off & On & On \\
\hline 240 degree & On & On & On & On & Off & On \\
\hline 300 degree & On & On & On & On & On & Off \\
\hline
\end{tabular}

\section{Simulation And MeAsurement Results}

\section{A. Radiation Patterns}

Figure 5 presents the measurement results of the radiation pattern in two orthogonal planes when the antenna was fixed by the substrate of PATHE perpendicular to the ground and was facing to the linearly polarized reference antenna in E- and H-Plane respectively. The measured and simulated radiation patterns in two orthogonal planes for PATHE are shown in Fig. 5 (a) and (b). The radiation patterns at $60^{\circ}$ intervals in azimuth plane for six directions with the scale normalized to $0 \mathrm{~dB}$ are illustrated in Fig. (c). It can be seen from Fig.5 (a) and (b), the measured radiation patterns planes obtained in two orthogonal planes are matched with the simulated radiation pattern of an elevation view respectively. In addition, the measured radiation patterns in the orthogonal are similar. This indicates the PATHE is circularly polarized. The measured beamwidth from Fig.5 (a) and (b) is approximately $50^{\circ}$.

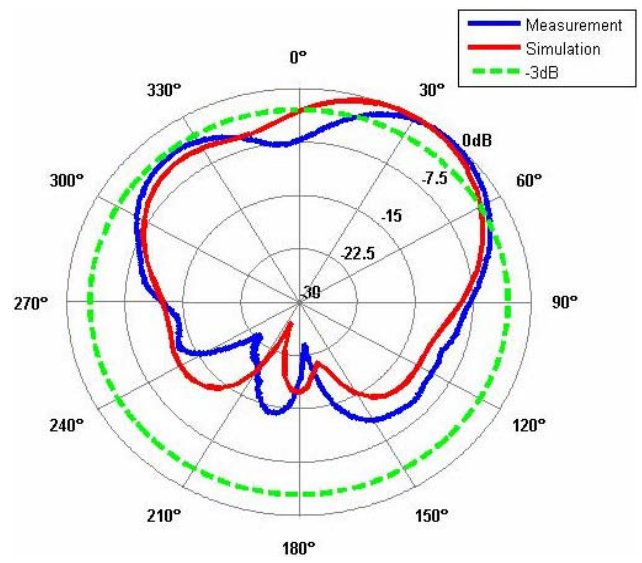

(a) The radiation pattern when the reference antenna in E-Plane,

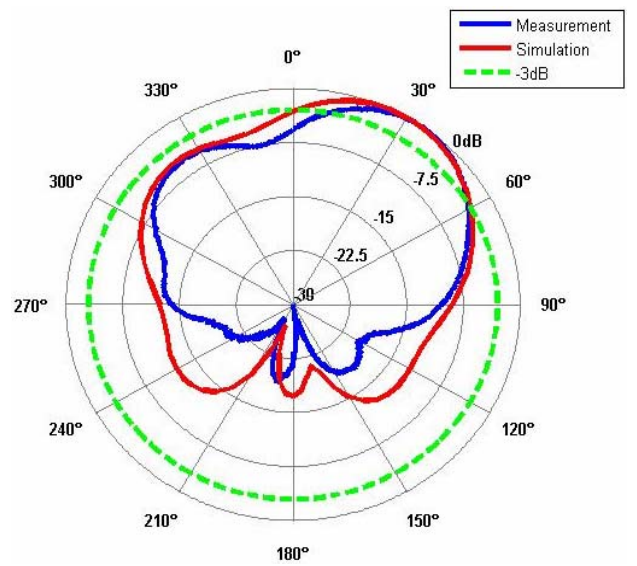

(b) The radiation pattern when the reference antenna in H-plane,

Fig.5. Comparison of the simulated and measured radiation pattern in elevation plane for PATHE when the reference antenna is in E-and H-Plane.

The $3 \mathrm{~dB}$ beamwidth obtained from the simulation is about $60^{\circ}$. A detailed comparison is given in Table II where the single-feed PATHE has a larger impedance bandwidth of approximately $7.5 \%$. According to the switching table, radiation patterns at $60^{\circ}$ intervals in the azimuth plane for six directions can be obtained as shown in Fig. 6. 
TABLE II

Comparison of Measured and Simulated Resonant Frequency, REFLECTION COEFFICIENT AND BANDWIDTH FOR PATHE

\begin{tabular}{|l|c|c|c|}
\hline Results & $\begin{array}{c}\text { Resonant } \\
\text { Frequency (GHz) }\end{array}$ & $\begin{array}{c}\mathbf{S}_{\mathbf{1 1}} \\
\mathbf{( d B})\end{array}$ & $\begin{array}{c}\text { 10dB } \\
\text { Bandwidth } \\
\text { (MHz) }\end{array}$ \\
\hline \multirow{2}{*}{ Measured } & 2.520 (Max.) & -14.8 & 180 \\
\cline { 2 - 4 } & 2.440 (Second Max.) & -14.3 & 180 \\
\hline \multirow{2}{*}{ Simulated } & 2.440 (Max.) & -17.2 & 160 \\
\cline { 2 - 4 } & 2.500 (Second Max.) & -14.6 & 160 \\
\hline
\end{tabular}

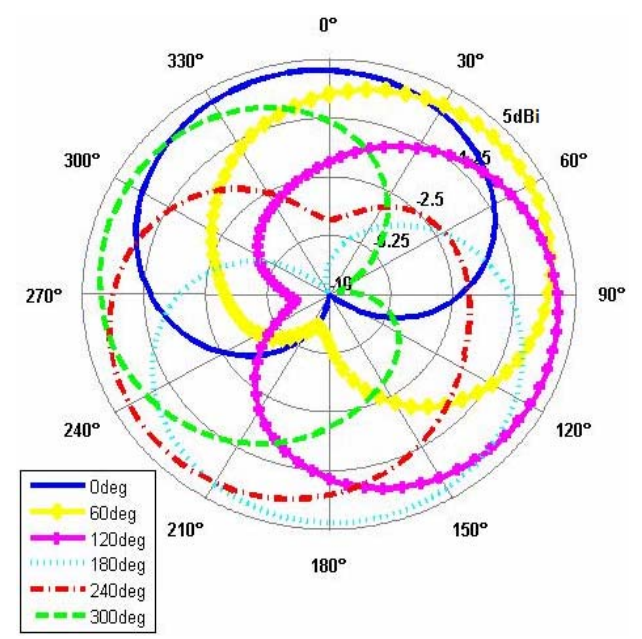

Fig. 6 Radiation patters at $60^{\circ}$ intervals in azimuth plane for six directions

\section{B. Reflection Coefficients and Frequency Bandwidth}

The following parameters were obtained from simulation results, where the front to back ratio is around $20 \mathrm{~dB}$ at $60^{\circ}$, whilst it is about $5.09 \mathrm{~dB}$ in azimuth plane, and the peak gain of this antenna is $5.56 \mathrm{dBi}$ with the elevation angle around $60^{\circ}$ when the main beam lobe directs to a specific orientation in azimuth plane. The reflection coefficient, $\mathrm{S}_{11}$, as a function of frequency was obtained from both simulation and measurement as shown in Fig. 7. The simulated and measured results for $\mathrm{S}_{11}$ are similar. Table III indicates that the $10 \mathrm{~dB}$ bandwidth of PATHE is $>180 \mathrm{MHz}(7.5 \%)$, which is much larger than the one obtained from PASHE (140MHz).

TABLE III

Comparison of Measured and Simulated Reflection Coefficient and BANDwidTh For Pashe ANd PATHe

\begin{tabular}{|c|c|c|c|c|}
\hline \multirow{2}{*}{ Results } & \multicolumn{2}{|c|}{ PASHE } & \multicolumn{2}{c|}{ PATHE } \\
\cline { 2 - 5 } & Measured & Simulated & Measured & Simulated \\
\hline $\begin{array}{c}\text { Reflection } \\
\text { Coefficient } \\
(\mathrm{dB})\end{array}$ & -35.00 & -19.64 & $-14.8 /-14.3$ & $\begin{array}{c}-17.2 /- \\
14.6\end{array}$ \\
\hline $\begin{array}{c}10 \mathrm{~dB} \\
\text { Bandwidth } \\
(\mathrm{MHz})\end{array}$ & 140 & 140 & 180 & 160 \\
\hline
\end{tabular}

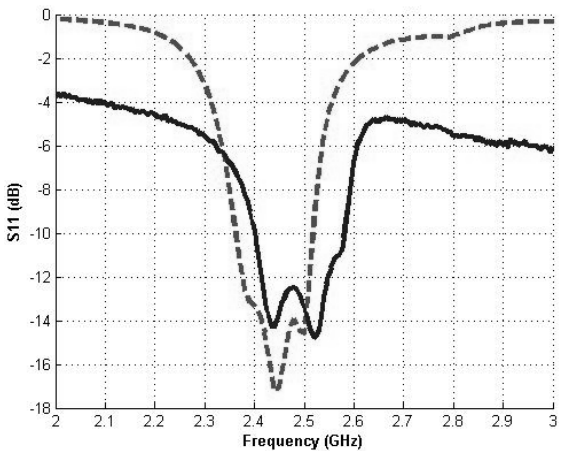

Fig. 7 Comparison of measured and simulated reflection coefficients.

\section{CONCLUSIONS}

A single-feed smart patch antenna array with circularly polarized wave, (PATHE) was designed, constructed and verified through simulation and measurement. The reflection coefficient as a function of frequency obtained from both simulation and measurement indicated that the single-feed PATHE has a larger frequency bandwidth of around $7.5 \%$, compared to previously reported antennas of similar construction. The improvement is due to improved edge effects and mutual coupling, and the additional elements placed in an outer circle. The patch antenna array with a hexagonal shape element provided a much wider frequency bandwidth. This overcomes the inherent problems of narrow bandwidth of traditional microstrip patch antennas. This smart patch antenna with high performances, low profile, and a simple beam-controlling circuit can meet the demand for increasing indoor/outdoor mobile wireless terminals.

\section{REFERENCES}

[1] R. Schlub, J. Lu and T. Ohira, "Seven element ground skirt monopole ESPAR antenna design from a genetic algorithm and the finite element method," IEEE Transactions on Antennas and Propagation, vol. 51, no. 11, pp. 3033-3039, Nov. 2003

[2] J. Lu, D. Ireland, and R. Schlub, "Dielectric embedded ESPAR (DEESPAR) antenna array for wireless communications," IEEE Transactions on Antennas and Propagation, vol. 53, no. 8, pp. 2437 2443, Aug. 2005.

[3] D. Gray, J. Lu, and D. Thiel, "Electronically steerable Yagi-Uda microstrip patch antenna array," IEEE Transactions on Antennas and Propagation, vol. 46, no. 5, pp. 605-608, May 1998.

[4] D. Thiel and S. Smith, Switched Parasitic Antennas for Cellular Communications, Norwood, MA, Artech House, Inc., 2001.

[5] M. Shi, J. Lu and D. Ireland, "Smart Patch Antenna for Indoor Mobile Wireless Computing," Proceedings of APMC-2005, vol. 3, pp 19131916, Dec. 2005.

[6] Jui-Han Lu and Kai-Ping Yang, "A simple design for single-feed circularly-polarized microstrip antennas," Proc. Natl. Sci. Counc ROC (A), Vol. 24, No. 2, pp 130-133, 2000.

[7] HFSS v9.0. (2004). Ansoft Corporation [Online]. Available: http://www.ansoft.com/products/hf/hfss/

[8] C. Balanis, “Antenna Theory Analysis and Design", pp 871, $3^{\text {rd }}$ Edition, John Wiley and Sons Inc, New Jersey, 2005. 